\title{
Process Automation in Computer Controlled Polishing
}

\author{
David Walker ${ }^{1,2,3 a *}$, Guoyu Yu, ${ }^{1, b}$, Caroline Gray,c, Paul Rees ${ }^{1, d}$, \\ Matt Bibby ${ }^{2, ~ e}$, Hsing-Yu Wu ${ }^{1,3 f}$, Xiao Zheng ${ }^{1, g}$
}

\author{
${ }^{1}$ National Facility For Ultra Precision Surfaces, OpTIC-Glyndwr, Fford William Morgan, St. Asaph \\ Business Park, St Asaph, North Wales LL17 0JD, UK \\ ${ }^{2}$ Zeeko Ltd, Vulcan Court, Vulcan Rd, Hermitage Industrial Estate, Coalville, LE67 3FW, UK \\ ${ }^{3}$ University College London, Glower St, London, WC1E 6BT, UK \\ addwlkr@aol.com bguoyuyu@yahoo.commail cc.gray@glyndwr.ac.uk dp.rees@glyndwr.ac.uk \\ ématt.bibby@zeeko.co.uk fandy810301@gmail.com ${ }^{9}$ zxiao0629@gmail.com
}

Keywords: Optics, Mirror, Lens, Polishing, Smoothing, Grolishing, Automation, Telescope, Robot

\begin{abstract}
We report on an ambitious project in the field of automation, applied to bound and free abrasive processing of precision and ultra-precision surfaces, with potentially far-reaching consequences. This involves two main aspects:- direct processing of surfaces using industrial robots, and combining robots with Zeeko CNC polishing machines to automate operations that are currently manual. These form steps towards our ultimate vision of the Integrated Manufacturing Cell for bespoke optics rather than mass-produced, and manufacture of other precision surfaces including prosthetic joint implants. Projects such as the European Extremely Large Telescope provide a relevant case study, where significant numbers of high-value bespoke optics are required.
\end{abstract}

\section{Introduction}

The European Southern Observatory (ESO) is building a 39m optical/near-infrared telescope, to be located at 3060m altitude at Cerro Amozenes in Chile [1]. The primary mirror will comprise $798 \mathrm{x}$ $1.4 \mathrm{~m}$ off-axis aspheric, hexagonal mirror segments (6-off $\times 133$ different hexagonal shapes and offaxis forms), and a further 133 spares (one of each design), giving a total requirement of 931 segments. The prototype segment specification [2] (briefly summarised in Table 1) is challenging, not least because the segments must match in base radius and conic constant to create a holistic $39 \mathrm{~m}$ mirror. The required quality and quantity presents an unprecedented challenge to optical manufacture. A single bespoke mirror of this type might take 18 months or more to procure, but EELT demands 2-3 per week. ESO therefore commissioned two organisations to manufacture fullsize prototypes, and we first report on the work of one - the National Facility for Ultra Precision Surfaces at OpTIC in North Wales, collaborating with Zeeko Ltd.

Table 1 E-ELT Prototype Segment Specification

\begin{tabular}{|l|l|l|}
\hline \multicolumn{1}{|c|}{ Simplified surface specification } & Average & \multicolumn{1}{c|}{ Max. } \\
\hline RMS form (excluding 10mm edge-zone) & $25 \mathrm{~nm}$ & $50 \mathrm{~nm}$ \\
\hline RMS form (Zernike allowances removed representing warping harnesses) & $7.5 \mathrm{~nm}$ & $15 \mathrm{~nm}$ \\
\hline PVq (95\%) mis-figure in edge-zone around hexagon & $100 \mathrm{~nm}$ & $200 \mathrm{~nm}$ \\
\hline
\end{tabular}

A $200 \mathrm{~mm}$ thick and highly stable, $1.5 \mathrm{~m}$ a/c hexagonal, Master Spherical Segment (MSS) was polished on the Zeeko IRP1600 machine at OpTIC. It achieved $18.6 \mathrm{~nm}$ RMS and 4-5nm rms at intermediate spatial frequencies [3] as required for differential metrology of the thin and flexible aspheric segments with respect to the Master. Three off-axis aspheric segments were ground on the Cranfield University BoX machine ${ }^{\mathrm{TM}}[4]$ and then polished on the IRP1600. 


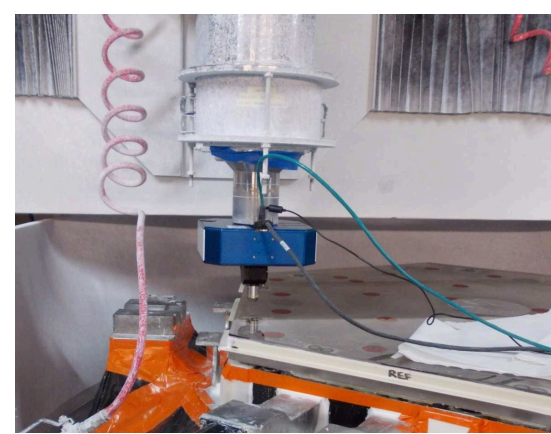

Fig. 1 STA1 measuring a segment
In-process metrology and certification used full-aperture nulling interferometry with respect to the MSS. This was complemented by sub-aperture interferometry to stitch over CGH artefacts and a small central obstruction. Independent verification was provided by autocollimator/pentaprism profilometry, and texture was measured using a 4D Technologies STA1 white light interferometer, Fig. 1. All metrology was conducted in-situ on the Zeeko machine. The segments have been accepted by ESO, and the metrology facility is unique in having been certified by ESO as compliant. A fourth aspheric segment is in process.

Beyond E-ELT segments, there is burgeoning demand for large optics in several sectors - telescope optics and instrumentation, high-power laser systems, photolithography, space-optics, synchrotron optics etc. In addressing this demand, we are building on the experience gained in the segment project in two new ways - speeding up the total process chain, and process-automation.

\section{Speeding up the Process Chain for Metre-scale Optics}

Comparison of Platforms The IRP1600 at OpTIC, is statically and dynamically stiff. Like others of capacity $\geq 300 \mathrm{~mm}$, a granite base carries both the C-axis turntable (part horizontal), and the granite bridge which moves in $\mathrm{X}$ on parallel rails. The $\mathrm{Y}$ carriage moves along the bridge and supports a massive $\mathrm{Z}$ stage, and thence the $\mathrm{A} \& \mathrm{~B}$ axes which adjust the inclination of the integrated H-axis tool-spindle. A \& B are configured to provide a 'virtual pivot', located at the centre of the spherical polishing bonnet. This ensures that the centre of the polishing spot does not move on the part, as the $\mathrm{H}$-axis is inclined to follow the local surface-normal, or a specified angular offset of the $\mathrm{H}$-axis to the local normal at the part ('precession offset'). The maximum XYZ feed-rates for the IRP1600 are $0.1 \mathrm{~m} / \mathrm{s}$, with absolute positional accuracy of 20 microns. This is well-matched to bonnet-polishing, where $\mathrm{X} \& \mathrm{Y}$ define the tool-path, and $\mathrm{Z}$ is programmed to follow a complex surface, and to compress the bonnet against the surface to define the polishing spot-sizes (which can be varied 'on the fly'). The innate stability of the machine also lends itself to on-machine metrology, giving an integrated polishing and metrology capability.

A $3.05 \mathrm{~m}$ reach, $125 \mathrm{Kg}$ payload Fanuc robot, also at OpTIC, can process optics of roughly the same size as the Zeeko machine. The robot is a much lighter-weight structure, and is based on a series of six rotational axes in a cantilevered design. The positional performance is much inferior to the Zeeko machine at only $+/ 0.2 \mathrm{~mm}$ repeatability (not absolute accuracy), the stiffness considerably lower, and there is no integrated tool-spindle or $\mathrm{C}$-axis to rotate the part. However, motion-speeds are much higher, the three principle motions (as used for executing a tool-path) providing up to 1.92 radians/second; equivalent to a lateral traverse speed of nearly $4 \mathrm{~m} / \mathrm{s}$ at a typical $2 \mathrm{~m}$ reach.

The platforms are therefore complementary:- the IRP1600 delivers high stiffness, absolute 3D positional accuracy and integrated $\mathrm{C}$ and $\mathrm{H}$ axes. The machine lends itself to slow-motion processes mediated by precise 3D positioning on complex surfaces (such as bonnet polishing of aspheres and free-forms, including treatment of edges and perforations), and to on-machine metrology. The robot is well-suited to processes where traverse-speed rather than precise positioning is the principle concern. In particular, larger tooling will be inherently less sensitive to XY positional errors, and tooling which 'floats' on the surface of the part under gravity is insensitive to $\mathrm{Z}$ errors.

What has become clear from the segment project is that the time for the Zeeko process to meet specification is critically dependent upon the input quality from CNC grinding. In particular, mid spatial frequency defects, in the form of annular ripples, are time-consuming to remove, as are 
global form errors in the 10's of microns regime (probably originating in grinding support issues). This has led us to consider the suitability of the robot for a versatile intermediate smoothing and possibly corrective process, between $\mathrm{CNC}$ grinding and corrective polishing on the Zeeko machine.

Toolpath Software With this in view, the kinematics and control of Fanuc and ABB robots have been incorporated within the standard Zeeko TPG (Tool Path Generator) software suite. This enables standard or developmental tool-paths designed in TPG (including raster, crossed-raster, spiral, adaptive spiral, and the special pseudo-random unicursal toolpath [5], to be output to a Zeeko machine or Fanuc/ABB robot at will. Moreover, given metrology data, the full corrective capability of the Zeeko software (feed-rate moderation or Precessions ${ }^{\mathrm{TM}}$ numerical optimisation), can seamlessly be deployed on a robot.

Robot Grolishing We use the term 'grolishing' to describe a family of processes between grinding and polishing, and have previously reported on such processes deployed on Zeeko machines [6]. The objective is to remove mid spatial frequency defects from grinding and, ultimately, conduct the first stage of form correction using profilometer metrology feedback. However, contamination of the Zeeko machine with coarse (and particularly diamond) abrasives is a concern, as this could potentially degrade texture or scratch/dig achieved in subsequent fine polishing. With this in view, we report progress porting grolishing processes onto robot platforms using the extended Zeeko TPG software, and describe our preliminary results below. Fig. 2 shows a general view of the main Robot $\mathrm{Lab}$ at OpTIC. The $125 \mathrm{Kg}$ payload Fanuc robot is at the front, and the $40 \mathrm{Kg}$ payload ABB behind.

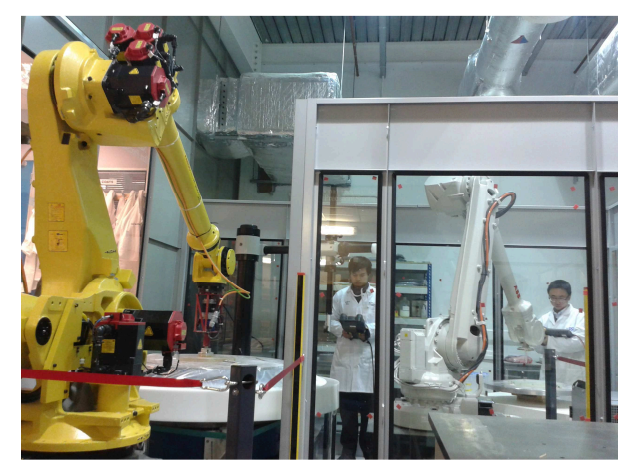

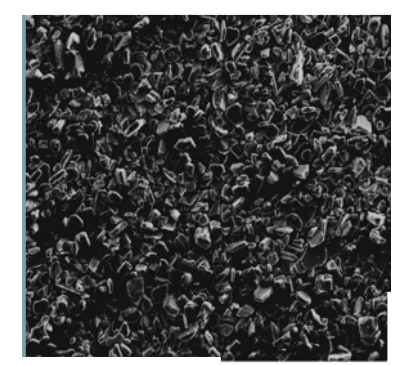
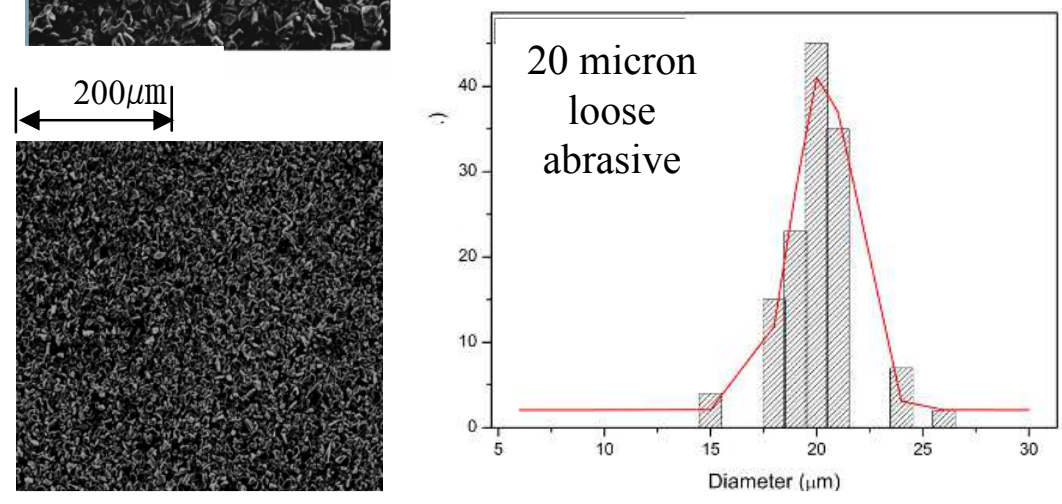

Fig. 4

Photomicrographs of $20 \mu \mathrm{m}$ (top) and $9 \mu \mathrm{m}$ (bottom) aluminium oxide abrasives

A series of parametric removal trials has been conducted with various tooling mounted in an articulated interface. An examples is given of a $100 \mathrm{~mm}$ diameter brass tool (Fig. 3), working a $400 \mathrm{~mm}$ across-corners hexagon, of pre-polished borosilicate glass. Considerable experience has 
been accumulated using hard tooling and cerium oxide polishing compound to smooth aspheric segments, without introducing new mid spatial frequency artefacts. From this, $100 \mathrm{~mm}$ was considered a safe (minimum) diameter as a baseline for developing the robot-grolishing process with larger abrasive sizes. Two abrasives ( 9 and 20 micron aluminium oxide), two tool-speeds (600 and $1000 \mathrm{rpm}$ ) and two tool-loads (1020 and 1370 grams) were used in the specific trials reported here. For each of the eight combinations, three experiments were performed, in order to define the consistency of results, as shown in Table 2.

Table 2 Robot-grolishing parameters and removal / texture results

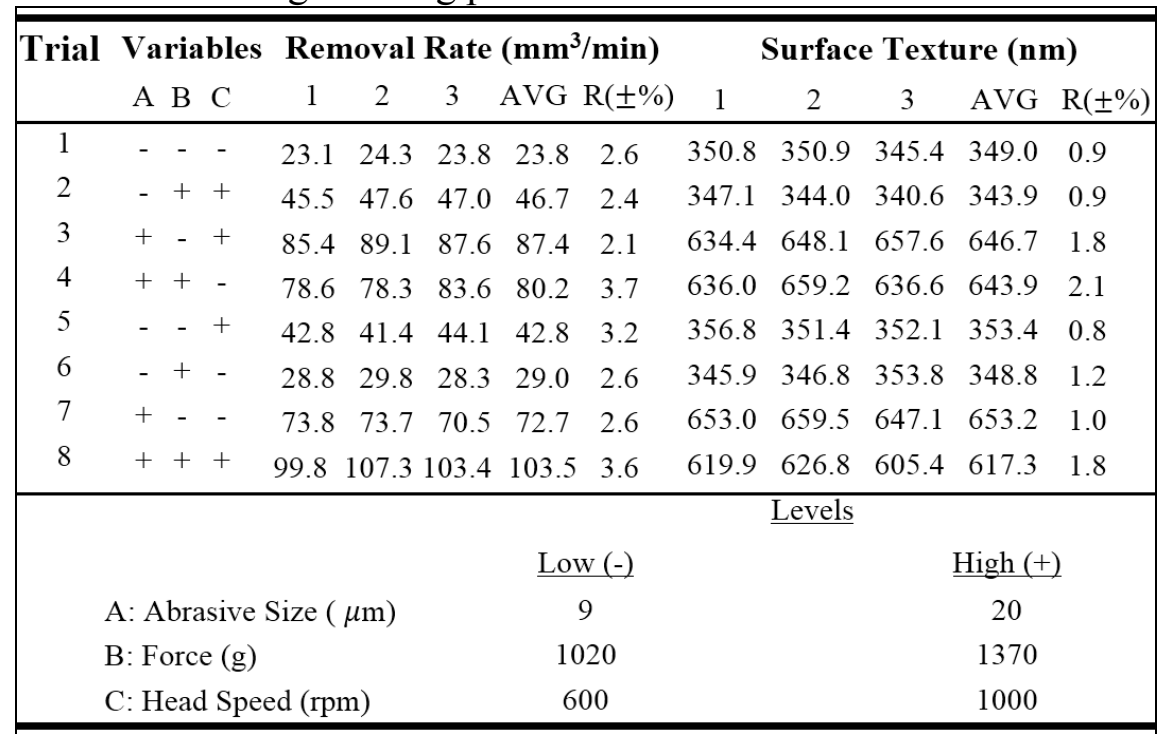

Table 3 Robot-grolishing parameters and edge results

T F A H Edge (E)-to-Edge(F) Corner(C)-to-Corner(D)

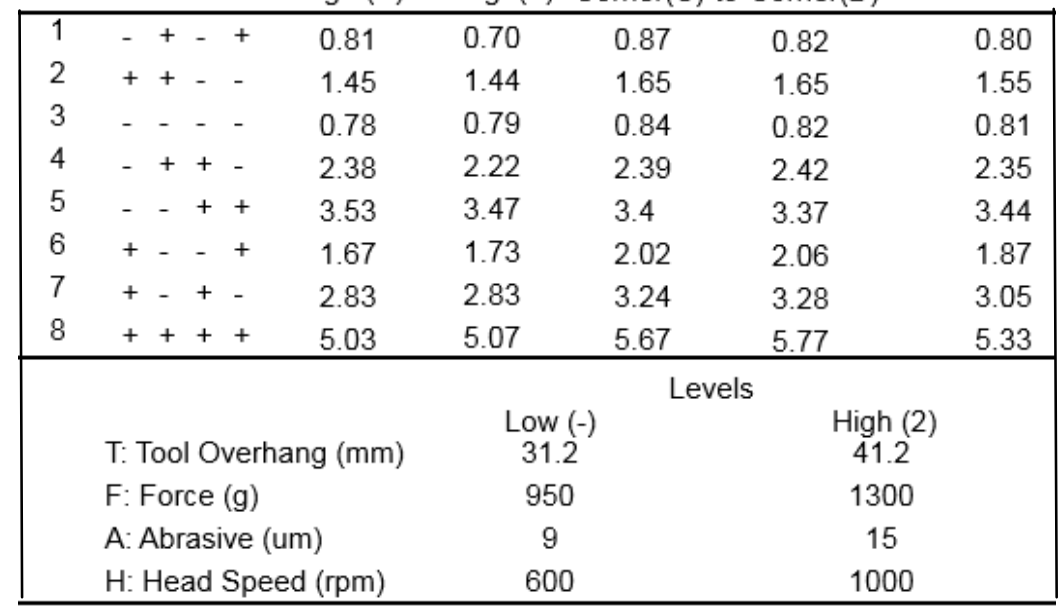

Volumetric removal rate was near-linear with abrasive, force and head-speed, and repeatability about $+/-3 \%$. Similarly, surface texture was primarily dependent on only the abrasive used.

Photomicrograph images of grains, and particle-size distributions from imageanalysis, are presented in Figs. 4 and 5 respectively, and confirm a tail longwards of the peak, which will influence texture.

Parametric trials were also conducted to examine how edge-profiles can be controlled using the robot platform. The parameters are defined in Table 3, and edgeresults presented in Fig. 6 . This confirms that it is possible to programme edges to be gently turned up by a controlled amount, providing a surface-profile that lends itself to corrective bonnetpolishing on a Zeeko machine.
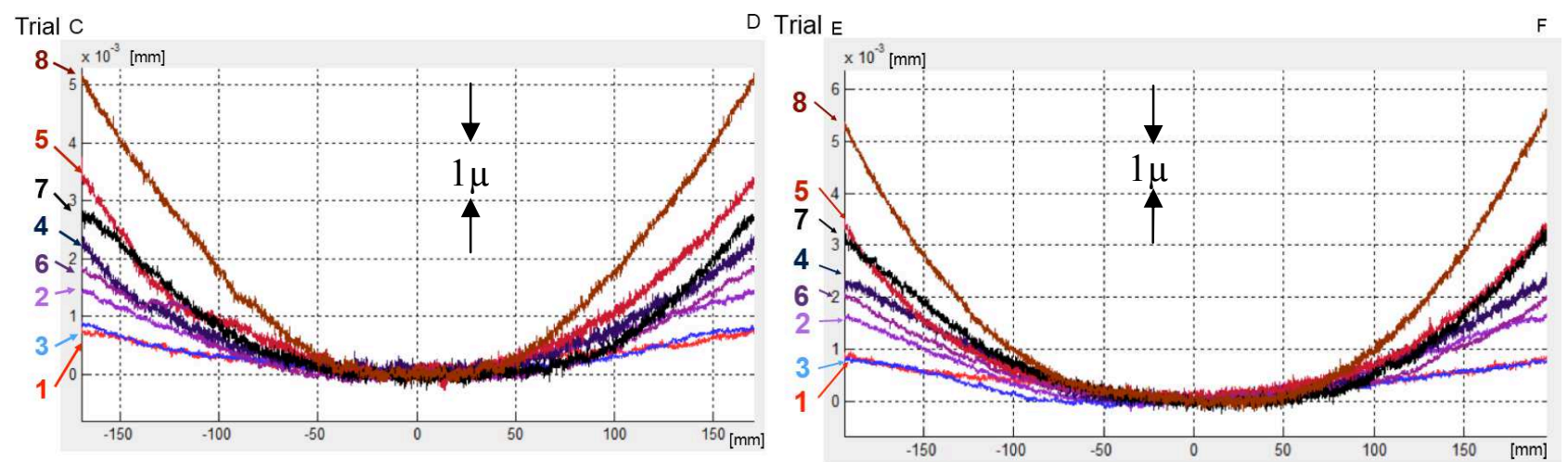

Fig. 6 Removal profiles on 400mm hexagon; across sides (left); across corners (right) 


\section{Preliminary results with bound-diamond pads}

We have very recently embarked on developing an alternative smoothing approach, using annular flexible diamond pads, mounted via a compliant layer to give ability to adapt to an aspheric profile. Such tooling can address more severe aspheres than the hard brass tools used with aluminium oxide. This work draws on the development of the Shape Adaptive Grinding (SAG) process by Beaucamp et.al. [7], but extending to larger pads rather than bonnets, and deployed on a robot. The new tooling is mounted via the articulation mechanism, on the rotating spindle, and water provides cooling. First trials on $400 \mathrm{~mm}$ borosilicate a/c hexagons are shown in Table 4 and Fig. 7, using extremely aggressive $250 \mu$ diamond, and also the more benign $20 \mu$ diamond (which can be compared with the C20 results above). The parts were prepared using C9 loose abrasive, and texture was measured

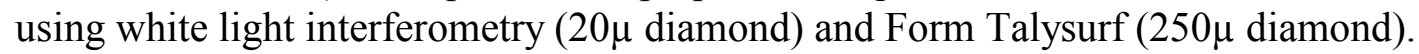
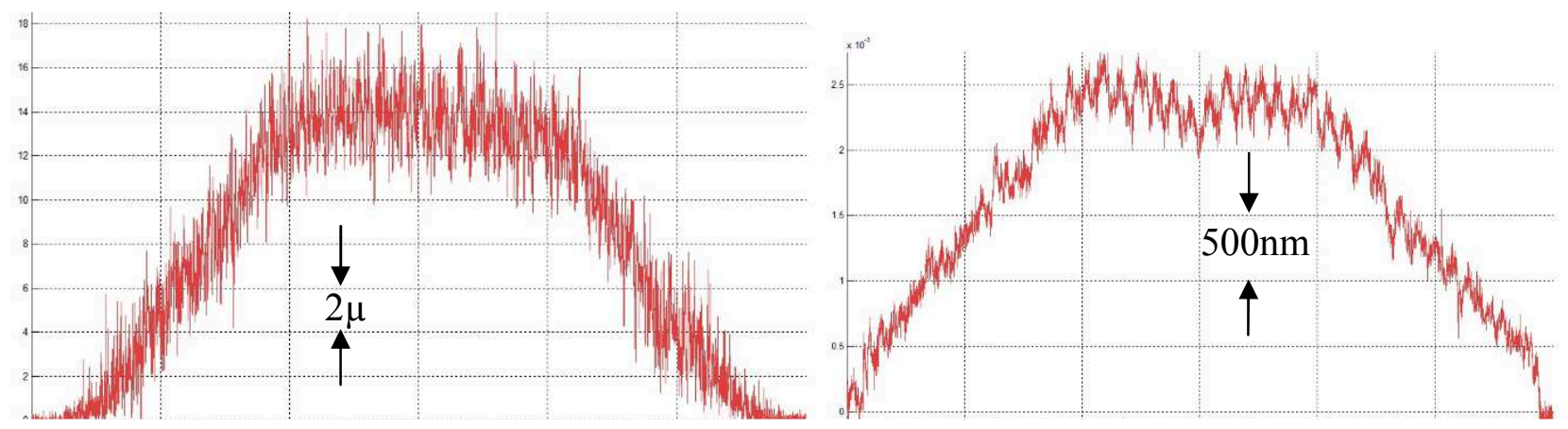

Fig. 7 Removal profiles with 250 micron diamond pad (left) and 20 micron (right) 200rpm spindle speed, applied force $8.2 \mathrm{KgF}$, time $2 \mathrm{~m} 30 \mathrm{~s}$

Table 4 Preliminary results using KGS bound-diamond abrasive pads

\begin{tabular}{|l|c|c|}
\hline Abrasive & 250 micron bound diamond & 20 micron bound diamond \\
\hline Vol. removal rate & $267 \mathrm{~mm}^{3} / \mathrm{min}$ & $45 \mathrm{~mm}^{3} / \mathrm{min}$ \\
\hline Texture $\mathrm{nm}$ & $1150 \mathrm{~nm} \mathrm{Sa}$ & $392 \mathrm{~nm} \mathrm{Sa}$ \\
\hline
\end{tabular}

\section{Process Automation}

Many activities such as part and tool loading/unloading, changing between polishing and metrology, and transport between different processing and metrology stations are typically performed manually. For optics at the metre scale, these tasks, followed by realignment of the part, can occupy substantial time, and demonstrably increase risk of damage. We have already automated the alignments required for full or sub-aperture interferometry, avoiding the need for human intervention. Automation of the intermediate processes that are still manual, would therefore present the opportunity for full end-to-end process automation, creating a closed manufacturing cell. This is the direction of current work, and embraces both on-machine metrology, and the use of a separate Metrology Station. The work is driven both by the need to support metre-scale optics fabrication, and by specific industry requirements at the small mass-produced optics end of the market.

First, a Fanuc M20i-A robot has been positioned adjacent to a Zeeko IRP600 machine in the Robot Lab at OpTIC. A third, XYZ CNC system is used to simulate a grinder or metrology station, allowing prototyping of cell operation. This has required the development of a control system, which allows co-ordination of movement of three separate CNC systems. This application is being developed using a combination of Matlab, for rapid prototyping, and a new user interface developed using C\#, called Zeeko Studio. It is planned that future systems could incorporate a CNC grinding machine, as well as robot pre-polishing station and Zeeko machine. 


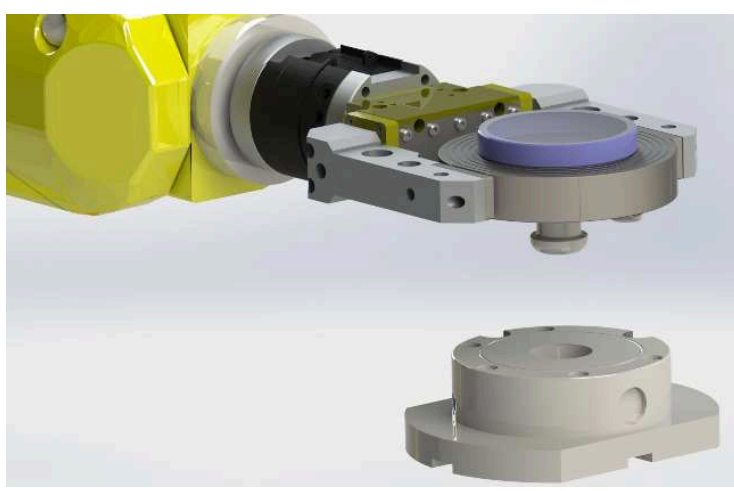

Fig. 8 CAD rendering of M20i-A robot loading a part into a holder on the IRP600 machine

\section{Conclusion}

Ultimately, we envisage a decision making application to control activities of the cell and allow production to a specification input by the user. Instead of controlling the system via individual interfaces with each component machine, the user will interface only with a cell control terminal. The robot arm will move components within the cell as well as perform processing and change tools. Current status is that parts for auto-interchange are out for manufacture, and the CAD model can be seen in Fig. 8. The authors expect limited cell operation to be demonstrable summer 2015

We have taken important steps towards the ultimate goal of the integrated and automated manufacturing cell for precision and ultra-precision surfaces. Most notably, we have demonstrated preliminary work in robot smoothing using fixed and loose abrasives, with the robots under direct and very versatile control of an extended version of the Zeeko Tool Path Generator software suite. These processes are intended in part as intermediate steps between CNC grinding, and CNC polishing on a Zeeko machine; or, for less precise components in their own right. We are also progressing using a robot to automate currently manual operations on $\mathrm{CNC}$ machines and expect to demonstrate this in the coming months. The overall aim is to optimise deployment of process-steps between robot and Zeeko machine, or other CNC platforms, in an integrated Cell.

\section{Acknowledgements}

The authors acknowledge EPSRC, Innovate-UK, STFC and the Welsh Government funding for aspects of this research. The assistance of W. Lu of Imperial College London in obtaining SEM images of abrasives, and deriving particle size distributions, is greatly appreciated. Hsing-Yu Wu acknowledges an MOE Technologies Incubation Scholarship, Ministry of Education, Taiwan.

\section{References}

[1] R. Gilmozzi, J. Spyromilio, The European Extremely Large Telescope (E-ELT), The Messenger, Vol. 127, 2007

[2] E. Swat, ESO prototype segment specification, E-SPE-ESO-300-0150 Issue 4, 1-33, (2009)

[3] D. Walker, I. Baker, R. Evans, S. Hamidi, P. Harris, H. Li, W. Messelink, J. Mitchell, M. ParryJones, P. Rees, G. Yu, Proceedings SPIE, Vol. 8126, doi: 10.1117/12.893360

[4] Comley P, Morantz P., Shore P and Tonnellier X, CIRP Annals - Manufacturing Technology, 60(1), 379-382 (2011)

[5] C. R. Dunn, D. D. Walker, Optics Express, published by Optical Society of America on http://www.opticsexpress.org/, Vol. 16 Issue 23, 2008, pp.18942-18949

[6] D.D. Walker, A. Baldwin, R. Evans, R. Freeman, S. Hamidi, P. Shore, X. Tonnellier, S. Wei, C. Williams, G. Yu, Proc. SPIE Optical Manufacturing and Testing Conference, 2007, 07/2008; DOI: $10.1117 / 12.787930$

[7] A. Beaucamp, Y. Namba' H. Combrinck, P. Charlton, R. Freeman, CIRP Annals Manufacturing Technology, Vol. 63(1), pp 317-320 (2014) 